\title{
REVIEW
}

\section{The critical role of autophagy in plant responses to abiotic stresses}

\author{
Yu WANG ${ }^{1}$, Jie ZHOU $(\bowtie)^{1,2}$, Jingquan YU ${ }^{1,2,3}$ \\ 1 College of Agriculture and Biotechnology, Zhejiang University, Hangzhou 310058, China \\ 2 Zhejiang Provincial Key Laboratory of Horticultural Plant Integrative Biology, Hangzhou 310058, China \\ 3 Key Laboratory of Horticultural Plants Growth, Development and Quality Improvement, Ministry of Agriculture, \\ Hangzhou 310058, China
}

\begin{abstract}
Autophagy is an evolutionary conserved recycling process in eukaryotes whereby intracellular components are engulfed by autophagosomes, which are subsequently transferred to the vacuoles for further degradation and reuse. In organisms like yeast and metazoans, autophagy is actively engaged during environmental perturbation either by degrading denatured proteins and organelles or by interfacing with stress related signaling molecules. Studies over the last decade have also revealed numerous important mechanisms where autophagy is widely involved in plant abiotic stress responses. Autophagy serves as a pivotal route for nutrient remobilization by the degradation of superfluous or damaged cellular cytoplasmic material and organelles. It is also reported to regulate the accumulation of reactive oxygen species, to maintain the cellular redox balance of plants under stressful conditions. Furthermore, autophagy is essential in regulating cellular toxicity by removing aggregated and/or denatured proteins and thereby improving plant stress tolerance. In this review, recent advances in our understanding of autophagy, along with pathways and regulatory networks through which it influences many aspects of plant growth and development in response to nutrient starvation, oxidative stress, osmotic stress and extreme temperatures are discussed.
\end{abstract}

Keywords abiotic stresses, autophagy, extreme temperature, nutrient starvation, osmotic stress, oxidative stress
Received November 7, 2016; accepted December 19, 2016

Correspondence: jie@zju.edu.cn

\section{Introduction}

Autophagy is an evolutionary conserved degradation process in eukaryotes that breaks down cellular components for recycling during developmental transitions as well as under stress conditions ${ }^{[1,2]}$. In organisms, autophagy can be divided into microautophagy ${ }^{[3]}$, macroautophagy ${ }^{[4]}$, chaperone-mediated autophagy ${ }^{[5]}$, based on the specific process and mechanism, while types of organelle-specific autophagy are classified according to the specificity of substrates ${ }^{[6]}$. In plants, microautophagy and macroautophagy have been reported. Microautophagy occurs by invagination of the tonoplast into vacuoles ${ }^{[3]}$. Basic research on autophagy in eukaryotes has primarily focused on macroautophagy, hereafter referred to as autophagy. In plants, autophagy is initiated by formation of double-membrane vesicles called autophagosomes which engulf the intracellular contents. Afterwards, the outer membrane of autophagosome fuses with the vacuolar membrane, forming a single-membrane structure known as an autophagic body, which is degraded by hydrolases in the vacuole ${ }^{[7,8]}$.

Autophagy is executed by autophagy-related $(A T G)$ genes. The first $A T G$ was discovered in yeast (Saccharomyces cerevisiae); subsequently many ATGs were identified in different plant species, including Arabidopsis, tobacco (Nicotiana tabacum), rice (Oryza sativa), tomato (Solanum lycopersicum), wheat (Triticum dicoccoides), maize (Zea mays), and apple (Malus domestica) based upon their sequence similarity with yeast $A T G s^{[1,9,10]}$. In plants, more than $30 A T G$ genes have been identified and grouped depending on the intensive functional investigation of their counterparts in yeast, such as the Atg1/13 kinase complex; the vacuolar-protein-sorting (VPS)-34 phosphatidylinositol 3-kinase complex; the Atg9/2/18 transmembrane complex and the entire Atg8/12 
conjugation system ${ }^{[1,11]}$. The process of autophagy is regulated by TOR (targets of rapamycin) kinase signaling $^{[12]}$. TOR prevents integration of Atg 1 and Atg13 via phosphorylation of Atg13. When TOR is inhibited, Atg13 can associate with Atg1, which then promotes phosphorylation and triggers autophagy ${ }^{[13]}$. Phosphatidylinositol 3-kinase complexes, including VPS34, VPS15 and Atg6/ Beclin1, are essential for the induction of vesicles and nucleation $^{[14]}$. Atg9 is localized in the preautophagosomal structure (PAS) and non-PAS structures, and it is thought to deliver lipid to the forming autophagosomes ${ }^{[1,15]}$. The movement between PAS and non-PAS requires the participation of $\operatorname{Atg} 2$ and $\operatorname{Atg} 18$. The Atg8/12 ubiquitinlike conjugation systems mediate the expansion of the autophagosome membrane and cargo engulfment ${ }^{[15]}$. The C-terminal of Atg8 protein is first cleaved by Atg4, a cysteine protease, exposing a glycine residue, which then binds to an E1-like enzyme, $\operatorname{Atg} 7^{[16]}$. Latter, the Atg8 is transferred to Atg3, an E2-like enzyme. At last, the Atg8 is conjugated to the membrane lipid phosphatidylethanolamine (PE) to form an Atg8-PE complex, which can be cleaved by Atg4 for further recycling ${ }^{[1,14]}$. Similarly, Atg12 first binds with Atg7, is then transferred to another E2-like enzyme, Atg10, and finally conjugates with Atg5, forming an Atg12-Atg5 complex ${ }^{[17,18]}$. In yeast, Atg12Atg 5 further interacts with Atg16 $6^{[4]}$. An Atg16-like protein has been reported in Arabidopsis and maize ${ }^{[9,19]}$, but its function remains unclear in plants. Finally, the Atg8-PE conjugates and Atg12-Atg5 complexes promote autophagosomes expansion and movement toward vacuoles ${ }^{[20]}$.

The regulation of autophagy in yeast and animals has been thoroughly investigated ${ }^{[4,21]}$, but knowledge of this in a plant system still remains fragmentary. Use of RNA interference (RNAi) to reduce the expression of AtTOR results in an upregulated expression of some $A T G s$ and constitutive activation of autophagy, which is largely dependent on AtATG18a in Arabidopsis, indicating that TOR is an antagonistic regulator of autophagy in plants ${ }^{[22]}$. NBR1 (neighbor of the BRCA1 gene), the first isolated selective autophagy receptor with a ubiquitin-association domain in plants, interacts with both Atg8 and ubiquitin, and is involved in the integration of ubiquitinated protein aggregates for degradation through autophagy ${ }^{[23,24]}$. NBR1 is essential for plant tolerance to abiotic stresses, such as heat, oxidative, salt and drought stress, but has no function in age- and darkness-induced senescence and resistance to necrotrophic pathogen ${ }^{[23]}$. Furthermore, tomato HsfAla (heat shock transcription factor A1a) has a crucial role in drought tolerance by increasing the transcript levels of $A T G 10$ and $A T G 18 f$ coupled with induction of autophagosomes formation ${ }^{[25]}$. Atg18a interacts with WRKY33 transcription factor to mediate the formation of autophagosomes and resistance to necrotrophic pathogens, while silencing of WRKY33 compromises the tolerance to Botrytis cinerea ${ }^{[26,27]}$. In addition, reactive oxygen species (ROS) also mediate the activation of autophagy in plants ${ }^{[28]}$. Treatment with hydrogen peroxide in Arabidopsis, promotes the expression of autophagy-related genes and increases the number of autophagosomes $^{[29]}$. Autophagy is blocked by NADPH oxidase inhibitors during nutrient and salt stress, but not in osmotic stress ${ }^{[30]}$. Although the current understanding of regulation and mechanism of autophagy in plants is poor, it's operation in response to various environmental stresses has been investigated by genetic and molecular analyses of knockout and/or knockdown mutants ${ }^{[2,11,31]}$. Using the existing knowledge, this review provides an overview of recent advances in our understanding of the regulation of autophagy in plants as a response to diverse abiotic stresses.

\section{Role of autophagy in plants challenged with abiotic stresses}

\subsection{Nutrient deficiency and induced-senescence}

In plants, autophagosomes engulf cellular contents and transfer their cargo to the vacuole for degradation and reuse to meet the needs of cells under nutrient deficient conditions ${ }^{[32]}$. Sucrose starvation in suspension-cultured cells is used as a model system to analyze plant autophagy ${ }^{[8]}$. When tobacco suspension-cultured BY-2 cells are grown in a sucrose-free medium for $2 \mathrm{~d}$, more than $30 \%$ of the intracellular proteins are degraded ${ }^{[33]}$. In addition, the perinuclear regions of the cytosol of carbonstarved cells accumulate a number of small spherical bodies after treatment with the cysteine protease inhibitor E-64c for $8 h^{[33]}$. Takatsuka et al. investigated the role of 3-methyladenine, an inhibitor of autophagy in mammalian system, in tobacco culture cells under sucrose starvation and found that 3-methyladenine can inhibit the formation of autophagosomes even in the absence of sucrose ${ }^{[34]}$. Carbon starvation arrests cell growth and transiently induces the expression of AtATG3, AtATG4a, AtATG4b, AtATG7 and AtATG8 family genes and the formation of autophagosomes in Arabidopsis suspension-cultured cells at the onset of starvation ${ }^{[35]}$. Furthermore, under sucrose starvation conditions extensive autophagy can be triggered to degrade cytoplasmic constituents in rice suspension cells ${ }^{[36]}$. Thus, autophagy is necessary for cell survival during nutrient deficient conditions.

In Arabidopsis, $A T G$ defective mutants, such as atg2 and atg5, exhibit an early-senescence phenotype even under nutrient-rich conditions ${ }^{[37]}$ and the atg mutants are hypersensitive to nutrient-limiting conditions ${ }^{[38]}$. The atg 7 and atg9 plants display accelerated senescence in nitrogen- and carbon-free medium, characterized by premature chlorosis of the mature rosette leaves and a gradual decrease in chlorophyll content ${ }^{[18,39,40]}$. Additionally, plants lacking $A T G 13$ and $A T G 18 a$ are hypersensitive to nutrient deficiency and senesce, similar to other mutants 
missing components of the autophagy system ${ }^{[41,42]}$. Phillips et al. found that atg 10-1 mutated plants are more sensitive to nitrogen and carbon starvation than wild-type (WT), as characterized by elevated leaf senescence and programmed cell death (PCD), which are accompanied by failure to accumulate autophagosomes in the vacuole ${ }^{[18]}$. The rate of primary root elongation, total number of lateral roots per primary root and the total length of lateral roots per primary root in Atatg $4 a 4 b-1$ double mutant plants are remarkably low in the absence of nitrogen in comparison to WT ones, suggesting that autophagy is required for root elongation under nutrient starvation conditions ${ }^{[16]}$. In addition, Atg11 interacts with the Atg1/13 complex by promoting vesicle delivery to the vacuole and atg 11 mutant plants exhibit hypersensitivity to nitrogen and fixed-carbon limitations, and compromise the activation of general autophagy and senescence-induced mitophagy ${ }^{[43]}$. Therefore, autophagy is involved in regulating nutrient utilization and plant development in the absence of nitrogen or carbon. Other organisms show a similar phenotype to Arabidopsis under nutrient starvation conditions. The maize atg12 mutants show inhibited growth, elevated leaf senescence and compromised autophagic bodies formation during nitrogen or fixed-carbon starvation ${ }^{[9]}$. Starvation dramatically induces the expression of $A T G 8 i$ in soybean $^{[44]}$. Pérez-Pérez et al. investigated the molecular characterization of autophagy in Chlamydomonas reinhardtii and demonstrated that cell aging and nutrient limitation could increase the abundance of $\operatorname{Atg} 8^{[45]}$. On carbon or nitrogen starvation medium, Physcomitrella atg 5 mutants display an early-senescence phenotype compared with WT, which is due to the lack of cytoplasmic degradation by autophagy leading to amino acid imbalance ${ }^{[46]}$. These results suggest that autophagy plays a critical role in response to nutrient stress for nitrogen and carbon mobilization.

Autophagy is a conserved process of bulk degradation, and nutrients recycling in all eukaryotes. Autophagy can selectively target plant organelles, such as chloroplasts and endoplasmic reticulum (ER), and their contents broken down by autophagic bodies, which is vital not only in plant development but also in response to various environmental stresses $^{[47]}$. Wada et al. identified the role of autophagy disruption in affecting the number and size of chloroplasts during senescence under dark ${ }^{[48]}$. The number and size of chloroplasts in individually darkened leaves of WT plants decreased, but this was compromised in atg $4 a 4 b-1$ plants ${ }^{[48]}$. Furthermore, while chloroplast stroma can be transported into the vacuole by Rubisco-containing bodies (RCBs), a specialized type of autophagic vesicle, this process is not observed in the vacuoles of atg mutant plants ${ }^{[49-51]}$. The RCBs are detected in rice during energy limitation, while they are inhibited in the presence of an inhibitor of autophagy or in an $A T G 7$ knockout mutant ${ }^{[50]}$. Tunicamycin or dithiothreitol induces ER stress-activated autophagy in Arabidopsis root cells, and fragments of ER can be engulfed by autophagosomes for degradation in vacuoles, a process mediated by inositol-requiring enzyme $1 \mathrm{~b}$, one of the ER stress sensors ${ }^{[47]}$. Taken together, upon nutrient starvation, the activity of TOR is inhibited which subsequently promotes Atg13 association with Atg1 and Atg11 to trigger autophagy for engulfing cellular components, including mitochondria, RCBs and fragments of ER, which are then transferred to the vacuole for degradation by hydrolases for nutrient reuse (Fig. 1).

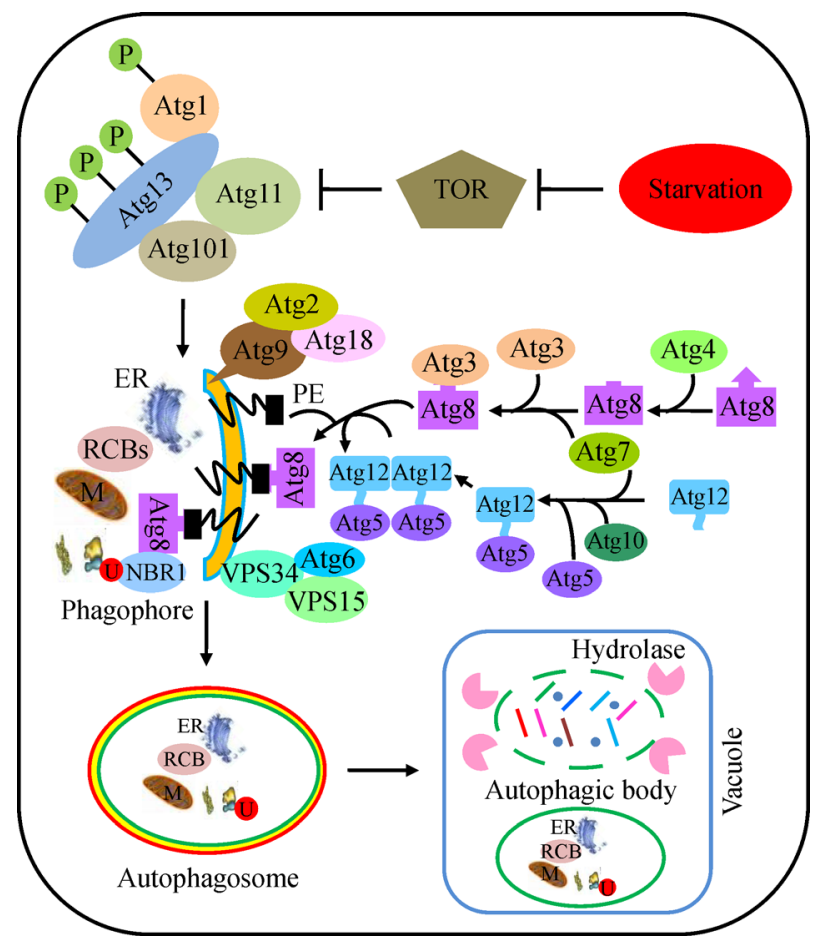

Fig. 1 Schematic representation of induction of autophagy by nutrient starvation in plants. When plants are exposed to deprived nutrient conditions, the activity of TOR is inhibited and subsequently promotes Atg13 association with Atg1 and Atg11 to induce formation of autophagy for engulfing cellular components including mitochondria, RCBs and fragments of ER, and then transfers them to the vacuole for degradation by hydrolases for nutrient reutilization. Atg, autophagy-related gene; P, phosphate group; TOR, targets of rapamycin; ER, endoplasmic reticulum; PE, phosphatidylethanolamine; RCBs, Rubisco-containing bodies; M, mitochondria; U, ubiquitin; NBR1, neighbor of the $B R C A 1$ gene; VPS, vacuolar-protein-sorting.

\subsection{Role in oxidative stress}

The production of ROS such as singlet oxygen, superoxide anion and $\mathrm{H}_{2} \mathrm{O}_{2}$ is increased during environmental stresses $^{[52]}$. In plants, ROS accumulation is controlled by the balance between production and scavenging through enzymatic and non-enzymatic mechanisms. The ROSscavenging enzymatic system contains vital enzymes such as ascorbate peroxidase, catalase, dehydroascorbate reductase, glutathione peroxidase, glutathione peroxiredoxin, glutathione reductase, monodehydroascorbate reductase, 
and superoxide dismutase. The systems of non-enzymatic scavengers include potent antioxidants, such as ascorbate and glutathione ${ }^{[53]}$. Under severe stress conditions, the ability to remove ROS through ROS-scavenging systems becomes weakened, resulting in excessive ROS accumulation, which leads to further oxidative stress and can cause damage to cellular components, including proteins, lipids and $\mathrm{DNA}^{[28]}$.

Numerous studies have reported that oxidative stress can induce autophagy both in animals and plants ${ }^{[28]}$. Autophagy-defective mutants of yeast accumulate higher levels of $\mathrm{H}_{2} \mathrm{O}_{2}$ than WT cells during nitrogen starvation conditions, and the excessive ROS can impair the respiratory pathway resulting in cell death ${ }^{[54]}$. These studies suggest that autophagy is essential for scavenging ROS and maintaining mitochondrial function under nutrient starvation. Treatment of Arabidopsis with $\mathrm{H}_{2} \mathrm{O}_{2}$ results in oxidation of proteins and induction of autophagy ${ }^{[29]}$. However, AtATG18a-RNAi plants are hypersensitive to $\mathrm{H}_{2} \mathrm{O}_{2}$ and accumulate more oxidized proteins than WT plants due to a lower rate of degradation by autophagy, indicating that autophagy is vital in removing degraded proteins under oxidative stress ${ }^{[29]}$. Consistent with these results, Yoshimoto et al. also demonstrated that atg2 and atg5 mutants accumulated higher levels of $\mathrm{H}_{2} \mathrm{O}_{2}$ compared to WT plants ${ }^{[37]}$. In addition to $\mathrm{H}_{2} \mathrm{O}_{2}$, treatment of Arabidopsis with methyl viologen, a kind of ROSproducing agent that receives electrons from PSI in chloroplasts and then reacts with oxygen to produce superoxide that is rapidly converted to $\mathrm{H}_{2} \mathrm{O}_{2}$, also activates autophagy ${ }^{[29]}$. Thus, ROS can activate autophagy, which in turn is important for the scavenging ROS-induced oxidized proteins. In addition, ER stress promotes the production of ROS and the accumulation of unfolded/misfolded proteins, leading to oxidative stress and induction of autophagy in singlet oxygen resistant1 mutant of Chlamydomonas reinhardtii ${ }^{[55]}$. Carotenoid-deficient mutants have low photoprotection ability, which results in high levels of ROS in the chloroplast, triggering autophagy ${ }^{[56]}$. Recent research has shown that submergence and ethanol treatments enhance the levels of ROS produced by NADPH oxidase in the rosettes of atg mutants, which is required for adaptation to submergence and ethanol stress, and activation of autophagy ${ }^{[57]}$. Inhibition of NADPH oxidase activity significantly suppressed the induction of autophagy by ROS, suggesting that NADPH oxidase induces production of $\mathrm{H}_{2} \mathrm{O}_{2}$ and has a partial role in triggering autophagy ${ }^{[56]}$. Zhang et al. found that cadmium stress induced the production of ROS that might act as a secondary messenger to promote the formation of autophagosomes ${ }^{[58]}$. In close agreement, atg mutants are more sensitive and exhibit more serious damages, such as higher oxidative damage and more ROS accumulation than WT plants during aluminum stress ${ }^{[59]}$. Silencing of glyceraldehyde-3-phosphate dehydrogenase (GAPDH), an important enzyme in energy metabolism, results in
ROS accumulation and activation of autophagy through inhibition of the interaction of Atg3 with GAPDH ${ }^{[60,61]}$. Interestingly, melatonin strongly increases the formation of autophagosomes under oxidative stress in Arabidopsis seedlings ${ }^{[62]}$. Moreover, rice atg $10 b$ mutants are hypersensitive to methyl viologen and accumulate high levels of oxidized proteins ${ }^{[63]}$. nbrl mutant plants also show compromised resistance to oxidative stress, like atg 5 and $\operatorname{atg} 7$ autophagy-defective plants ${ }^{[23]}$. This implies that autophagy plays a critical role in plant adaptation to oxidative stress by degrading oxidized proteins.

\subsection{Role in osmotic stress}

Osmotic stress such as salt and drought stress is the most common environmental stress that severely affects plant growth and development ${ }^{[64]}$. Globally, more than $6 \%$ of arable land and $30 \%$ of irrigated areas are subject to salinity-related problems ${ }^{[64]}$. With the change of global climate, the area of both salinity- and drought-prone areas will be increased in the future. Although salt stress and drought stress have some common features they are differently regulated in plants. Salt stress acts as both an ionic and osmotic stress for plants ${ }^{[65]}$, whereas osmotic stress enhances the accumulation of ROS, causing membrane disruption, enzyme dysfunction and oxidation of proteins ${ }^{[11]}$. As described above, autophagy plays an important role in scavenging oxidized proteins, indicating that autophagy might be involved in the response to osmotic stress.

Autophagy is identified as mediating enhanced tolerance to salt and drought stress. The expression of AtATG18a and the formation of autophagosomes are induced under both salt and drought conditions in Arabidopsis ${ }^{[30]}$. The growth of WT and RNAi-ATG18a seedlings is inhibited after salt and drought treatment, but this inhibition is more severe for RNAi-ATG18a plants, which display a chlorosis phenotype with salt application and high level of anthocyanin under drought stress ${ }^{[30]}$. In addition, induction of autophagy blocks ROS formation by applying an NADPH oxidase inhibitor during salt and drought stress ${ }^{[30]}$. NADPH oxidase inhibition can suppress the activation of autophagy under salt stress, but it has no effect on autophagy under drought stress ${ }^{[30]}$. These results indicate that autophagy is regulated by NADPH oxidasedependent and independent pathways. Recently, it was found that drought can upregulate numerous $A T G$ genes and promote autophagosomes formation in tomato ${ }^{[25]}$. HsfAla-silenced plants are sensitive, while the overexpressing (OE) plants are more tolerant toward drought stress. Drought stress increases the levels of insoluble proteins in HsfAla-silenced plants, and is associated with downregulation of $A T G 10$ and $A T G 18 f$ and decreased formation of autophagosomes ${ }^{[25]}$. Furthermore, electrophoretic mobility shift assay (EMSA) and chromatin immuno-precipitation coupled with qPCR (ChIP-qPCR) 
analysis reveal that HsfAla can directly bind to the promoters of $A T G 10$ and $A T G 18 f$. Silencing of $A T G 10$ and $A T G 18 f$ in WT and HsfAla OE plants compromises autophagosome formation and HsfAla-induced drought tolerance ${ }^{[25]}$. Thus, HsfA1a upregulates $A T G s$ expression and autophagosomes formation increases drought tolerance. Similarly, drought stress significantly increases the transcript level of $A T G S$ and autophagosome formation in wheat, rice and apple ${ }^{[10,66,67]}$. Li et al. analyzed the role of SiATG8a in foxtail millet (Setaria italica) in response to drought and nitrogen starvation ${ }^{[68]}$. Consistent with other research, the expression of SiATG8a is dramatically induced under drought stress $^{[68]}$ and overexpression of SiATG8a in Arabidopsis increases survival rates, chlorophyll content, and proline content when compared with WT plants ${ }^{[68]}$. In summary, salt and drought stress induce the expression of $A T G$ genes and promote the formation of autophagosomes to remove denatured proteins and impaired organelles, which results in enhanced stress tolerance.

\subsection{Role in extreme temperatures}

As with other abiotic stresses, extreme temperatures, such as heat and cold, impair plant growth and development ${ }^{[69]}$. Exposure to heat or cold results in the accumulation of insoluble and oxidized proteins ${ }^{[23,70]}$, which in turn induce autophagy. Yang et al. showed that heat stress activates autophagy resulting from accumulation of unfolded proteins in ER that in turn contribute to ER stress ${ }^{[71]}$. In Arabidopsis, atg5, atg 7 and nbrl mutants increase electrolyte leakage, but decrease the values of maximum quantum yield of PSII $(\mathrm{Fv} / \mathrm{Fm})$ during heat stress, indicating that the membrane integrity and the capacity of PSII photochemistry of atg and $n b r l$ mutants are more sensitive than WT plant under heat stress ${ }^{[23]}$. Moreover, the atg and $n b r l$ mutants accumulate enhanced levels of insoluble and detergent-resistant proteins that are highly ubiquitinated in comparison to WT plants under heat stress $^{[23]}$. Similarly, heat stress induces the expression of $A T G 5, A T G 7$ and NBR1 in tomato and the formation of autophagosomes $^{[72]}$. ATG5-, ATG7- and NBR1-silenced tomato plants show increased symptoms of heat shock, electrolyte leakage and suppress the formation of autophagosomes after heat treatment, suggesting that these knockdown plants are sensitive to heat stress ${ }^{[72]}$. In addition, both exogenous as well as endogenous melatonin enhances thermotolerance, which is associated with lowering the levels of insoluble and ubiquitinated proteins due to increased expression of heat-shock proteins, several $A T G$ genes and autophagosome formation to refold/ degrade denatured and unfolded proteins during heat shock $^{[73]}$. These results indicate that melatonin promotes protection of cellular proteins to enhance heat tolerance. Cheng et al. investigated the relationship between 2-Cys peroxiredoxins (2-CP) and autophagy during heat stress in tomato $^{[74]}$. Virus-induced gene silencing of tomato genes $2-C P 1,2-C P 2$, and $2-C P 1 / 2$ results in compromised heat tolerance, but increases the expression of ATG5 and $A T G 7$ and the formation of autophagosomes ${ }^{[74]}$. Likewise, $A T G 5$ - and $A T G 7$-silenced plants exhibit high levels of the transcript and increased abundance of 2-CP protein ${ }^{[74]}$. Thus, 2-CP interacts with autophagy to enhance tomato heat tolerance. Furthermore, the number of upregulated CaATG genes and autophagosomes in a pepper (Capsicum annuum) thermo tolerant variety was greater than in a thermo sensitive variety ${ }^{[75]}$. Cold stress also increases the transcript level of numerous $A T G$ genes and the formation of autophagosomes in pepper ${ }^{[75]}$. However, several ATGs are inhibited under cold stress in tobacco ${ }^{[76]}$. All these studies suggest that autophagy is actively involved in the plant's responses to extreme temperature stress.

\section{Conclusions and future perspectives}

Over recent decades, the identification of autophagyrelated genes and the vital roles of autophagy in the response to different abiotic stresses have been well established $^{[11,32]}$. In this review, we summarize the current research on and existing knowledge of the function of autophagy in plants compromised by abiotic stresses, such as nutrient starvation, oxidative stress, osmotic stress and extreme temperatures. A large number of studies demonstrate that transcription factors (TFs), stress responsive hormones, calcium ions and ROS have pivotal roles in plant responses to various abiotic and biotic stresses ${ }^{[77,78]}$. As shown in Fig. 2, the production of ROS increases under abiotic stresses and ROS-scavenging weakens, resulting in an impaired cellular redox balance due to excessive ROS accumulation, which further accelerates the accumulation of unfolded and oxidized proteins. Furthermore, abiotic stresses enhance the accumulation of denatured and unfolded proteins (Fig. 2). These damaged proteins trigger activation of autophagy for degradation of the denatured and unfolded proteins, thereby enhancing stress tolerance. In contrast, ROS generated by abiotic stresses may act as molecular signals to induce autophagy (Fig. 2). Stressinduced TFs, such as HsfAla, can directly bind to the promoters of $A T G$ genes to activate autophagy under environmentally perturbed conditions (Fig. 2). In addition, the activity of TOR is inhibited under nutrient starvation and the autophagy pathway is subsequently activated (Fig. 1).

Although the pivotal role of autophagy in the stress tolerance has been thoroughly investigated, the regulation and molecular mechanisms still remain poorly understood. Consequently, several important issues related to plant autophagy still need to be addressed. First, it was found that tomato HsfAla upregulates the expression of $A T G 10$ and $A T G 18 f$ and promotes autophagosome formation to increase drought tolerance ${ }^{[25]}$. Whether other TFs can also 


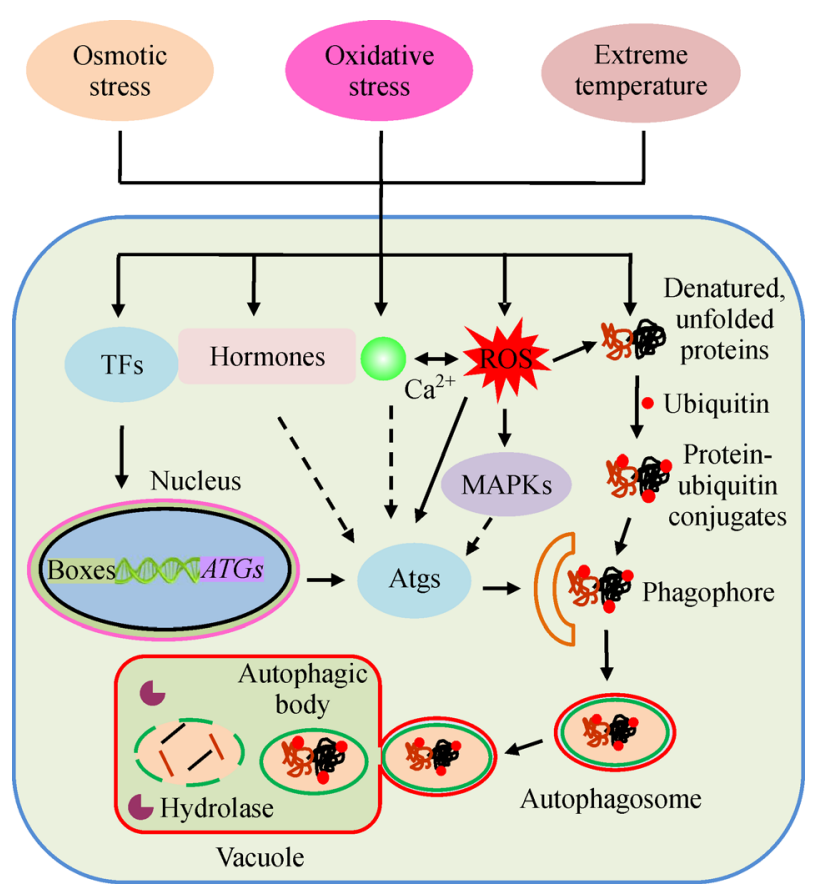

Fig. 2 A proposed model for the role and regulation of plant autophagy in response to osmotic, oxidative and extreme temperature stresses. Abiotic stresses, such as osmotic stress, oxidative stress and extreme temperature, increase the activity of TFs, the content of stress response hormones, the concentration of $\mathrm{Ca}^{2+}$ in the cytoplasm, the production of ROS and the accumulation of denatured and unfolded proteins. ROS can act as signaling molecules to activate autophagy, MAPKs and $\mathrm{Ca}^{2+}$, which in turn activates ROS. Excessive ROS further induces accumulation of oxidized proteins that are then ubiquitinated. In addition, TFs bind to the promoters of $A T G$ genes and directly regulate their expression to induce the formation of autophagosomes to engulf the ubiquitinated proteins for breakdown by hydrolase in the vacuole. The potential regulation of autophagy by hormones, $\mathrm{Ca}^{2+}$ and MAPKs is shown in this model. TFs, transcription factors; ATGs, autophagy-related genes; ROS, reactive oxygen species; MAPKs, mitogen-activated protein kinases.

regulate autophagic in responses in plants facing stresses, how these TFs regulate autophagy, and their possible functions in plant development and stress tolerance require further investigation. Second, $\mathrm{H}_{2} \mathrm{O}_{2}$ induced by NADPH oxidase increases the activation of c-Jun N-terminal kinase, the homologies of plant MAPKs (mitogenactivated protein kinases), which in turn promote the induction of autophagy in animal cells ${ }^{[79,80]}$. In plants, NADPH oxidase-dependent production of $\mathrm{H}_{2} \mathrm{O}_{2}$ can elevate the activity of MAPK $1 / 2^{[81,82]}$. The autophagyrelated protein sequences contain a large number of phosphorylation sites. Whether MAPKs interact with autophagy-related proteins and how the MAPKs regulate autophagy need to be addressed (Fig. 2). Third, calcium released from ER and lysosomes activates a $\mathrm{Ca}^{2+}$ signaling pathway to induce autophagy in animal cells ${ }^{[83,84]}$. The regulation of autophagy by $\mathrm{Ca}^{2+}$ in plants is still a matter of investigation (Fig. 2). Last, autophagy is regulated by hormones in development and disease resistance in animals ${ }^{[85,86]}$. Whether hormones are involved in the regulation of autophagy in plants (Fig. 2) and the mechanism also needs to be studied.

Acknowledgements This work was supported by the National Key Research and Development Program of China (2016YFD0201001) and the National Natural Science Foundation of China (31401877).

Compliance with ethics guidelines Yu Wang, Jie Zhou, and Jingquan Yu declare that they have no conflicts of interest or financial conflicts to disclose.

This article is a review and does not contain any studies with human or animal subjects performed by any of the authors.

\section{References}

1. Liu Y M, Bassham D C. Autophagy: pathways for self-eating in plant cells. Annual Review of Plant Biology, 2012, 63(1): 215-237

2. Michaeli S, Galili G, Genschik P, Fernie A R, Avin-Wittenberg T. Autophagy in plants - what's new on the menu? Trends in Plant Science, 2016, 21(2): 134-144

3. Mijaljica D, Prescott M, Devenish R J. Microautophagy in mammalian cells: revisiting a 40-year-old conundrum. Autophagy, 2011, 7(7): 673-682

4. Yang Z, Klionsky D J. An overview of the molecular mechanism of autophagy. Current Topics in Microbiology and Immunology, 2009, 335: 1-32

5. Orenstein S J, Cuervo A M. Chaperone-mediated autophagy: molecular mechanisms and physiological relevance. Seminars in Cell \& Developmental Biology, 2010, 21(7): 719-726

6. Reumann S, Voitsekhovskaja O, Lillo C. From signal transduction to autophagy of plant cell organelles: lessons from yeast and mammals and plant-specific features. Protoplasma, 2010, 247(3-4): 233-256

7. Hofius D, Schultz-Larsen T, Joensen J, Tsitsigiannis D I, Petersen N H, Mattsson O, Jorgensen L B, Jones J D, Mundy J, Petersen M. Autophagic components contribute to hypersensitive cell death in Arabidopsis. Cell, 2009, 137(4): 773-783

8. Bassham D C, Laporte M, Marty F, Moriyasu Y, Ohsumi Y, Olsen L J, Yoshimoto K. Autophagy in development and stress responses of plants. Autophagy, 2006, 2(1): 2-11

9. Li F Q, Chung T, Pennington J G, Federico M L, Kaeppler H F, Kaeppler S M, Otegui M S, Vierstra R D. Autophagic recycling plays a central role in maize nitrogen remobilization. Plant Cell, 2015, 27(5): 1389-1408

10. Wang $\mathrm{P}$, Sun $\mathrm{X}$, Jia $\mathrm{X}$, Wang $\mathrm{N}$, Gong $\mathrm{X} \mathrm{Q}$, Ma $\mathrm{F}$ W. Characterization of an autophagy-related gene Mdatg8i from apple. Frontiers in Plant Science, 2016, 7: 720

11. Han S J, Yu B J, Wang Y, Liu Y L. Role of plant autophagy in stress response. Protein \& Cell, 2011, 2(10): 784-791

12. Schmelzle T, Hall M N. TOR, a central controller of cell growth. Cell, 2000, 103(2): 253-262

13. Kamada Y, Funakoshi T, Shintani T, Nagano K, Ohsumi M, Ohsumi Y. Tor-mediated induction of autophagy via an Apg1 
protein kinase complex. Journal of Cell Biology, 2000, 150(6): $1507-1513$

14. Thompson A R, Vierstra R D. Autophagic recycling: lessons from yeast help define the process in plants. Current Opinion in Plant Biology, 2005, 8(2): 165-173

15. Xie Z P, Klionsky D J. Autophagosome formation: core machinery and adaptations. Nature Cell Biology, 2007, 9(10): 1102-1109

16. Yoshimoto K, Hanaoka H, Sato S, Kato T, Tabata S, Noda T, Ohsumi Y. Processing of ATG8s, ubiquitin-like proteins, and their deconjugation by ATG4s are essential for plant autophagy. Plant Cell, 2004, 16(11): 2967-2983

17. Thompson A R, Doelling J H, Suttangkakul A, Vierstra R D. Autophagic nutrient recycling in Arabidopsis directed by the ATG8 and ATG12 conjugation pathways. Plant Physiology, 2005, 138(4): 2097-2110

18. Phillips A R, Suttangkakul A, Vierstra R D. The ATG12conjugating enzyme ATG10 is essential for autophagic vesicle formation in Arabidopsis thaliana. Genetics, 2008, 178(3): 13391353

19. Yoshimoto K. Beginning to understand autophagy, an intracellular self-degradation system in plants. Plant \& Cell Physiology, 2012, 53 (8): 1355-1365

20. Kwon S, Park O K. Autophagy in plants. Journal of Plant Biology, 2008, 51(5): 313-320

21. Farré J C, Krick R, Subramani S, Thumm M. Turnover of organelles by autophagy in yeast. Current Opinion in Cell Biology, 2009, 21 (4): 522-530

22. Liu Y M, Bassham D C. TOR is a negative regulator of autophagy in Arabidopsis thaliana. PLoS One, 2010, 5(7): e11883

23. Zhou J, Wang J, Cheng Y, Chi Y J, Fan B F, Yu J Q, Chen Z X. NBR1-mediated selective autophagy targets insoluble ubiquitinated protein aggregates in plant stress responses. PLOS Genetics, 2013, 9 (1): e1003196

24. Zhou J, Zhang Y, Qi J X, Chi Y J, Fan B F, Yu J Q, Chen Z X. E3 ubiquitin ligase CHIP and NBR1-mediated selective autophagy protect additively against proteotoxicity in plant stress responses. PLOS Genetics, 2014, 10(1): e1004116

25. Wang Y, Cai S Y, Yin L L, Shi K, Xia X J, Zhou Y H, Yu J Q, Zhou J. Tomato HsfA1 a plays a critical role in plant drought tolerance by activating $A T G$ genes and inducing autophagy. Autophagy, 2015, 11 (11): 2033-2047

26. Lai Z B, Wang F, Zheng Z Y, Fan B F, Chen Z X. A critical role of autophagy in plant resistance to necrotrophic fungal pathogens. Plant Journal, 2011, 66(6): 953-968

27. Zhou J, Wang J, Zheng Z Y, Fan B F, Yu J Q, Chen Z X. Characterization of the promoter and extended $\mathrm{C}$-terminal domain of Arabidopsis WRKY33 and functional analysis of tomato WRKY33 homologues in plant stress responses. Journal of Experimental Botany, 2015, 66(15): 4567-4583

28. Perez-Perez M E, Lemaire S D, Crespo J L. Reactive oxygen species and autophagy in plants and algae. Plant Physiology, 2012, 160(1): 156-164

29. Xiong Y, Contento A L, Nguyen P Q, Bassham D C. Degradation of oxidized proteins by autophagy during oxidative stress in Arabidopsis. Plant Physiology, 2007, 143(1): 291-299

30. Liu Y M, Xiong Y, Bassham D C. Autophagy is required for tolerance of drought and salt stress in plants. Autophagy, 2009, 5(7): 954-963

31. Zhou J, Yu J Q, Chen Z X. The perplexing role of autophagy in plant innate immune responses. Molecular Plant Pathology, 2014, 15(6): $637-645$

32. Bassham D C. Plant autophagy-more than a starvation response. Current Opinion in Plant Biology, 2007, 10(6): 587-593

33. Moriyasu Y, Ohsumi Y. Autophagy in tobacco suspension-cultured cells in response to sucrose starvation. Plant Physiology, 1996, 111 (4): 1233-1241

34. Takatsuka C, Inoue Y, Matsuoka K, Moriyasu Y. 3-methyladenine inhibits autophagy in tobacco culture cells under sucrose starvation conditions. Plant \& Cell Physiology, 2004, 45(3): 265-274

35. Rose T L, Bonneau L, Der C, Marty-Mazars D, Marty F. Starvationinduced expression of autophagy-related genes in Arabidopsis. Biology of the Cell, 2006, 98(1): 53-67

36. Chen M H, Liu L F, Chen Y R, Wu H K, Yu S M. Expression of $\alpha$ amylases, carbohydrate-metabolism, and autophagy in cultured rice cells is coordinately regulated by sugar nutrient. Plant Journal, 1994, 6(5): 625-636

37. Yoshimoto K, Jikumaru Y, Kamiya Y, Kusano M, Consonni C, Panstruga R, Ohsumi Y, Shirasu K. Autophagy negatively regulates cell death by controlling NPR1-dependent salicylic acid signaling during senescence and the innate immune response in Arabidopsis. Plant Cell, 2009, 21(9): 2914-2927

38. Chung T, Phillips A R, Vierstra R D. ATG8 lipidation and ATG8mediated autophagy in Arabidopsis require ATG12 expressed from the differentially controlled ATG12A and ATG12B loci. Plant Journal, 2010, 62(3): 483-493

39. Doelling J H, Walker J M, Friedman E M, Thompson A R, Vierstra $\mathrm{R} D$. The APG8/12-activating enzyme APG7 is required for proper nutrient recycling and senescence in Arabidopsis thaliana. Journal of Biological Chemistry, 2002, 277(36): 33105-33114

40. Hanaoka H, Noda T, Shirano Y, Kato T, Hayashi H, Shibata D, Tabata S, Ohsumi Y. Leaf senescence and starvation-induced chlorosis are accelerated by the disruption of an Arabidopsis autophagy gene. Plant Physiology, 2002, 129(3): 1181-1193

41. Suttangkakul A, Li F Q, Chung T, Vierstra R D. The ATG1/ATG13 protein kinase complex is both a regulator and a target of autophagic recycling in Arabidopsis. Plant Cell, 2011, 23(10): 3761-3779

42. Xiong Y, Contento A L, Bassham D C. AtATG18a is required for the formation of autophagosomes during nutrient stress and senescence in Arabidopsis thaliana. Plant Journal, 2005, 42(4): 535-546

43. Li F Q, Chung T, Vierstra R D. Autophagy-related11 plays a critical role in general autophagy- and senescence-induced mitophagy in Arabidopsis. Plant Cell, 2014, 26(2): 788-807

44. Htwe N, Tanigawa H, Ishibashi Y, Zheng S H, Yuasa T, IwayaInoue M. Nutrient starvation differentially regulates the autophagyrelated gene GmATG8i in soybean seedlings. Plant Biotechnology (Sheffield, England), 2009, 26(3): 317-326

45. Pérez-Pérez M E, Florencio F J, Crespo J L. Inhibition of target of rapamycin signaling and stress activate autophagy in Chlamydomonas reinhardtii. Plant Physiology, 2010, 152(4): 1874-1888

46. Mukae K, Inoue Y, Moriyasu Y. ATG5-knockout mutants of Physcomitrella provide a platform for analyzing the involvement of 
autophagy in senescence processes in plant cells. Plant Signaling \& Behavior, 2015, 10(11): e1086859

47. Liu Y M, Bassham D C. Degradation of the endoplasmic reticulum by autophagy in plants. Autophagy, 2013, 9(4): 622-623

48. Wada S, Ishida H, Izumi M, Yoshimoto K, Ohsumi Y, Mae T, Makino A. Autophagy plays a role in chloroplast degradation during senescence in individually darkened leaves. Plant Physiology, 2009, 149(2): 885-893

49. Izumi M, Hidema J, Ishida H. From Arabidopsis to cereal crops: conservation of chloroplast protein degradation by autophagy indicates its fundamental role in plant productivity. Plant Signaling \& Behavior, 2015, 10(11): e1101199

50. Izumi M, Hidema J, Wada S, Kondo E, Kurusu T, Kuchitsu K, Makino A, Ishida H. Establishment of monitoring methods for autophagy in rice reveals autophagic recycling of chloroplasts and root plastids during energy limitation. Plant Physiology, 2015, 167 (4): $1307-1320$

51. Izumi M, Wada S, Makino A, Ishida $H$. The autophagic degradation of chloroplasts via rubisco-containing bodies is specifically linked to leaf carbon status but not nitrogen status in Arabidopsis. Plant Physiology, 2010, 154(3): 1196-1209

52. Zhou J, Wang J, Li X, Xia X J, Zhou Y H, Shi K, Chen Z, Yu J Q H. $2 \mathrm{O}_{2}$ mediates the crosstalk of brassinosteroid and abscisic acid in tomato responses to heat and oxidative stresses. Journal of Experimental Botany, 2014, 65(15): 4371-4383

53. Foyer $\mathrm{C} \mathrm{H}$, Noctor G. Redox regulation in photosynthetic organisms: signaling, acclimation, and practical implications. Antioxidants \& Redox Signalling, 2009, 11(4): 861-905

54. Suzuki S W, Onodera J, Ohsumi Y. Starvation induced cell death in autophagy-defective yeast mutants is caused by mitochondria dysfunction. PLoS One, 2011, 6(2): e17412

55. Perez-Martin M, Perez-Perez M E, Lemaire S D, Crespo J L. Oxidative stress contributes to autophagy induction in response to endoplasmic reticulum stress in Chlamydomonas reinhardtii. Plant Physiology, 2014, 166(2): 997-1008

56. Pérez-Pérez M E, , Couso I, Crespo J L. Carotenoid deficiency triggers autophagy in the model green alga Chlamydomonas reinhardtii. Autophagy, 2012, 8(3): 376-388

57. Chen L, Liao B, Qi H, Xie L J, Huang L, Tan W J, Zhai N, Yuan L B, Zhou Y, Yu L J, Chen Q F, Shu W S, Xiao S. Autophagy contributes to regulation of the hypoxia response during submergence in Arabidopsis thaliana. Autophagy, 2015, 11(12): 2233-2246

58. Zhang W N, Chen W L. Autophagy induction upon reactive oxygen species in Cd-stressed Arabidopsis thaliana. San Francisco, CA. Proceedings of the Society for Photo-Instrumentation Engineers, 2010, 7568: 75681Y

59. Ren H, Li Y N, Zhao F F, Pu X J, Wei L J, Lv X, Zhu F, Lin H H. The role of autophagy in alleviating damage of aluminum stress in Arabidopsis thaliana. Plant Growth Regulation, 2016, 79(2): 167175

60. Han S J, Wang Y, Zheng X Y, Jia Q, Zhao J P, Bai F, Hong Y G, Liu Y L. Cytoplastic glyceraldehyde-3-phosphate dehydrogenases interact with ATG3 to negatively regulate autophagy and immunity in Nicotiana benthamiana. Plant Cell, 2015, 27(4): 1316-1331

61. Henry E, Fung N, Liu J, Drakakaki G, Coaker G. Beyond glycolysis: GAPDHs are multi-functional enzymes involved in regulation of ROS, autophagy, and plant immune responses. PLOS Genetics, 2015, 11(4): e1005199

62. Wang P, Sun X, Wang N, Tan D X, Ma F W. Melatonin enhances the occurrence of autophagy induced by oxidative stress in Arabidopsis seedlings. Journal of Pineal Research, 2015, 58(4): 479-489

63. Shin J H, Yoshimoto K, Ohsumi Y, Jeon J S, An G. OsATG10b, an autophagosome component, is needed for cell survival against oxidative stresses in rice. Molecules and Cells, 2009, 27(1): 67-74

64. Chaves M M, Flexas J, Pinheiro C. Photosynthesis under drought and salt stress: regulation mechanisms from whole plant to cell. Annals of Botany, 2009, 103(4): 551-560

65. Zhu J K. Cell signaling under salt, water and cold stresses. Current Opinion in Plant Biology, 2001, 4(5): 401-406

66. Kuzuoglu-Ozturk D, Yalcinkaya O C, Akpinar B A, Mitou G, Korkmaz G, Gozuacik D, Budak H. Autophagy-related gene, TdAtg8, in wild emmer wheat plays a role in drought and osmotic stress response. Planta, 2012, 236(4): 1081-1092

67. Rana R M, Dong S, Ali Z, Huang J, Zhang H S. Regulation of ATG6/Beclin-1 homologs by abiotic stresses and hormones in rice (Oryza sativa L.). Genetics and Molecular Research, 2012, 11(4): 3676-3687

68. Li W W, Chen M, Zhong L, Liu J M, Xu Z S, Li L C, Zhou Y B, Guo C H, Ma Y Z. Overexpression of the autophagy-related gene SiATG8a from foxtail millet (Setaria italica $\mathrm{L}$.) confers tolerance to both nitrogen starvation and drought stress in Arabidopsis. Biochemical and Biophysical Research Communications, 2015, 468(4): 800-806

69. Wang W X, Vinocur B, Altman A. Plant responses to drought, salinity and extreme temperatures: towards genetic engineering for stress tolerance. Planta, 2003, 218(1): 1-14

70. Wang F, Guo Z X, Li H Z, Wang M M, Onac E, Zhou J, Xia X J, Shi $\mathrm{K}$, Yu J Q, Zhou Y H. Phytochrome A and B function antagonistically to regulate cold tolerance via abscisic aciddependent jasmonate signaling. Plant Physiology, 2016, 170(1): 459-471

71. Yang X C, Srivastava R, Howell S H, Bassham D C. Activation of autophagy by unfolded proteins during endoplasmic reticulum stress. Plant Journal, 2016, 85(1): 83-95

72. Zhou J, Wang J, Yu J Q, Chen Z X. Role and regulation of autophagy in heat stress responses of tomato plants. Frontiers in Plant Science, 2014, 5: 174

73. Xu W, Cai S Y, Zhang Y, Wang Y, Ahammed G J, Xia X J, Shi K, Zhou Y H, Yu J Q, Reiter R J, Zhou J. Melatonin enhances thermotolerance by promoting cellular protein protection in tomato plants. Journal of Pineal Research, 2016, 61(4): 457-469

74. Cheng F, Yin L L, Zhou J, Xia X J, Shi K, Yu J Q, Zhou Y H, Foyer $\mathrm{C} \mathrm{H}$. Interactions between 2-Cys peroxiredoxins and ascorbate in autophagosome formation during the heat stress response in Solanum lycopersicum. Journal of Experimental Botany, 2016, 67 (6): 1919-1933

75. Zhai Y F, Guo M, Wang H, Lu J P, Liu J H, Zhang C, Gong Z H, Lu M H. Autophagy, a conserved mechanism for protein degradation, responds to heat, and other abiotic stresses in Capsicum annuum L. Frontiers in Plant Science, 2016, 7: 131

76. Zhou X M, Zhao P, Wang W, Zou J, Cheng T H, Peng X B, Sun M $\mathrm{X}$. A comprehensive, genome-wide analysis of autophagy-related 
genes identified in tobacco suggests a central role of autophagy in plant response to various environmental cues. DNA Research, 2015, 22(4): $245-257$

77. Xia X J, Zhou Y H, Shi K, Zhou J, Foyer C H, Yu J Q. Interplay between reactive oxygen species and hormones in the control of plant development and stress tolerance. Journal of Experimental Botany, 2015, 66(10): 2839-2856

78. Bose J, Pottosin I I, Shabala S S, Palmgren M G, Shabala S. Calcium efflux systems in stress signaling and adaptation in plants. Frontiers in Plant Science, 2011, 2: 85

79. Liu H, Zhang H, Iles K E, Rinna A, Merrill G, Yodoi J, Torres M, Forman H J. The ADP-stimulated NADPH oxidase activates the ASK-1/MKK4/JNK pathway in alveolar macrophages. Free Radical Research, 2006, 40(8): 865-874

80. Lorin S, Pierron G, Ryan K M, Codogno P, Djavaheri-Mergny M. Evidence for the interplay between JNK and p53-DRAM signalling pathways in the regulation of autophagy. Autophagy, 2010, 6(1): 153-154

81. Nie W F, Wang M M, Xia X J, Zhou Y H, Shi K, Chen Z X, Yu J Q.
Silencing of tomato $\mathrm{RBOH} 1$ and $M P K 2$ abolishes brassinosteroidinduced $\mathrm{H}_{2} \mathrm{O}_{2}$ generation and stress tolerance. Plant, Cell \& Environment, 2013, 36(4): 789-803

82. Zhou J, Xia X J, Zhou Y H, Shi K, Chen Z X, Yu J Q. RBOH1dependent $\mathrm{H}_{2} \mathrm{O}_{2}$ production and subsequent activation of MPK1/2 play an important role in acclimation-induced cross-tolerance in tomato. Journal of Experimental Botany, 2014, 65(2): 595-607

83. Crawford S E, Estes M K. Viroporin-mediated calcium-activated autophagy. Autophagy, 2013, 9(5): 797-798

84. Medina D L, Ballabio A. Lysosomal calcium regulates autophagy. Autophagy, 2015, 11(6): 970-971

85. Tian L, Ma L, Guo E E, Deng X J, Ma S Y, Xia Q Y, Cao Y, Li S. 20-hydroxyecdysone upregulates Atg genes to induce autophagy in the Bombyx fat body. Autophagy, 2013, 9(8): 1172-1187

86. Chen J Y, Wang L, Wu C, Hu Q, Gu C, Yan F, Li J R, Yan W, Chen G. Melatonin-enhanced autophagy protects against neural apoptosis via a mitochondrial pathway in early brain injury following a subarachnoid hemorrhage. Journal of Pineal Research, 2014, 56(1): $12-19$ 